\title{
Polyhydramnios and acute renal failure
}

\author{
D. V. Hamilton \\ M.R.C.P.
}

\author{
MoIRA B. KeLly \\ M.R.C.O.G.
}

\begin{abstract}
Summary
Acute renal failure secondary to ureteric obstruction is described in a primigravida with twin gestation and polyhydramnios. Relief of the obstruction occurred on drainage of the liquor and return to normal renal function following delivery.
\end{abstract}

\section{Introduction}

The usual causes of acute renal failure in pregnancy are hypotension, septicaemia, concealed accidental haemorrhage (abruptio placentae), or as part of the syndrome of pre-eclampsia. A patient is reported who developed acute renal failure due to obstructive uropathy secondary to polyhydramnios.

\section{Case report}

A 21-year-old Caucasian primigravida was admitted to the antenatal ward for rest, with a twin gestation at 26 weeks. The pregnancy had been uneventful until 16 weeks' gestation when twins were diagnosed. She had been anorexic for 2 days. On examination she was apyrexial, BP $130 / 80 \mathrm{mmHg}$, and a tense abdomen of term size with polyhydramnios. There was no peripheral oedema. Four days after admission oliguria was noted and this persisted despite i.v. fluids ( 2 litres $5 \%$ dextrose over $12 \mathrm{hr}$ ), and frusemide ( $250 \mathrm{mg}$ i.v.). At 11.50 p.m. on the 6th day after admission to hospital she was transferred to the Norfolk and Norwich Hospital: she had not felt any fetal movements for one day and complained of bilateral loin pain. On examination she was apyrexial, not dehydrated, BP 130/90 $\mathrm{mmHg}$ and a tachycardia of $120 / \mathrm{min}$. Her uterus was of term size and there was severe distension and oedema of her abdominal wall, and oedema of both legs to above the knees and oedema of the vulva. She was tender in both renal angles. At 1.35 a.m. on day 7 a Drew Smythe catheter was inserted and 5 litres of liquor amnii were drained, following which the uterus shrank to 26 weeks in size. Consequent upon the draingage of the liquor her urinary output improved and serum creatinine rapidly fell (Table 1 ). At 2.25 and 2.40 p.m. twin girls (stillborn, weighing 530 and $430 \mathrm{~g}$ ) were delivered: there was no macroscopic fetal abnormality detected. Within 5 days of delivery her serum creatinine was normal.

TABLE 1. Renal function of patient following admission to hospital

\begin{tabular}{cccc}
\hline Days after admission & Urea $(\mathrm{mmol} / \mathrm{l})$ & Creatinine $(\mu \mathrm{mol} / \mathrm{c}$ \\
\hline 5 & & 16.4 & 744 \\
6 & & 17.2 & 777 \\
7 & 1.00 a.m. & 18.8 & 640 \\
& 8.30 a.m. & 18.9 & 510 \\
8 & 3.30 p.m. & 15.7 & 300 \\
9 & & 14.6 & 152 \\
12 & & 9.4 & 91 \\
\hline
\end{tabular}

An i.v. urogram was performed 5 days post partum: the ureters were tortuous and dilated to the pelvic brim, but below this the ureters were of normal calibre (Fig. 1). There was bilateral hydronephrosis. A repeat urogram 3 months later was normal.

\section{Discussion}

Dilatation of the renal tracts is acknowledged to be almost universal in pregnancy with a few notable exceptions: those with pelvic kidneys or urinary diversions with ureters draining into ileal conduits have normal calibre to their ureters. It is now generally accepted that the cause of this dilatation is not hormonal as was previously supposed but due to ureteric obstruction by the gravid uterus at the pelvic brim (Roberts, 1976). The pelvic ureter is not dilated. Such 'obstruction' of the ureter is usually asymptomatic, although there have been isolated reports of acute hydronephrosis and renal colic in 


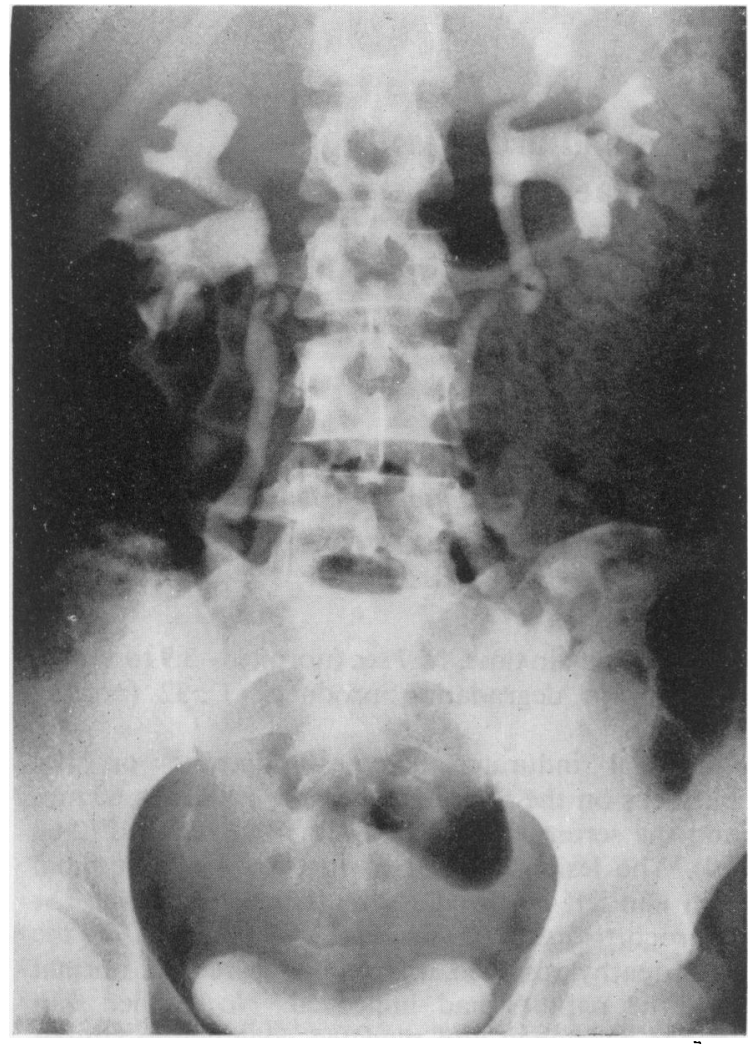

FIG. 1. Intravenous urogram, 5 days post partum, showing tortuous and dilated ureters above the pelvic brim, and bilateral hydronephrosis. Below the pelvic brim, the normal calibre of the right ureter is seen.

pregnancy secondary to acute ureteric obstruction which is relieved by positional change to the left lateral of knee-chest position (Schloss and Solomkin, 1952). Quigley and Cruikshank (1977) reported 2 patients with acute renal failure and polyhydramnios. In both patients, urine output and serum creatinine improved after amniocentesis and returned to normal following evacuation of the uterus. No i.v. urogram was performed in either patient.

O'Shaughnessy, Weprin and Zuspan (1980) have documented, with the use of ultrasonography, a case of acute renal failure at $\mathbf{3 4}$ weeks' gestation in a pregnancy complicated by polyhydramnios due solely to ureteral obstruction by an overdistended gravid uterus. Ultrasonography provides a useful means of diagnosing such patients (Fried, 1980).

Acute renal failure, secondary to ureteric obstruction, should be added to the causes of renal failure in pregnancies, particularly those complicated by polyhydramnios, especially if the accumulation of the fluid has been rapid. Patients with polyhydramnios should have careful assessment of renal function and should be advised to report if oliguria occurs.

\section{Acknowledgment}

We thank Mr R. E. Martin for permission to report this patient under his care.

\section{References}

FrIED, A.M. (1979) Hydronephrosis of pregnancy: Ultrasonographic study and classification of asymptomatic women. American Journal of Obstetrics and Gynecology, $135,1066$.

O'Shaughnessy, R., WePrin, S.A. \& ZuSPan, F.P. (1980) Obstructive renal failure by an overdistended pregnant uterus. Obstetrics and Gynecology, 55, 247.

Quigley, M.M. \& CRuikshank, D.P. (1977) Polyhydramnios and acute renal failure. Journal of Reproductive Medicine, 19, 92.

ROBERTS, J.A. (1976) Hydronephrosis of pregnancy. Urology, 8, 1.

Schloss, W.A. \& Solomkin, M. (1952) Acute hydronephrosis of pregnancy. Journal of Urology, 68, 885. 